\title{
Fenofibrate suppresses cellular metabolic memory of high glucose in diabetic retinopathy via a sirtuin 1-dependent signalling pathway
}

\author{
SHUZHI ZHAO ${ }^{1}$, JUN LI ${ }^{2}$, NA WANG ${ }^{1}$, BINGQING ZHENG ${ }^{1}$, \\ TAO $\mathrm{LI}^{1}, \mathrm{QING} \mathrm{GU}^{1}, \mathrm{XUN}^{\mathrm{X}} \mathrm{U}^{1}$ and $\mathrm{ZHI} \mathrm{ZHENG}^{1}$ \\ ${ }^{1}$ Department of Ophthalmology, First People's Hospital of Shanghai, Shanghai Jiaotong University, Shanghai 200080; \\ ${ }^{2}$ Department of Ophthalmology, Lishui City Center Hospital, Lishui, Zhejiang 323000, P.R. China
}

Received October 4, 2014; Accepted June 22, 2015

DOI: $10.3892 / \mathrm{mmr} .2015 .4164$

\begin{abstract}
Inflammation is a major contributing factor in the development of diabetic microvascular complications, regardless of whether improved glycaemic control is achieved. Studies have increasingly indicated that fenofibrate, a lipid-lowering therapeutic agent in clinical use, exerts a potential anti-inflammatory effect, which is mediated by sirtuin 1 (SIRT1; an NAD ${ }^{+}$-dependent deacetylase) in endothelial cells. The aim of the present study was to investigate the inhibitory effect of fenofibrate on metabolic memory (via the regulation of SIRT1), and inflammatory responses in cell and animal models of diabetic retinopathy (DR). The data demonstrated that high glucose treatment in human retinal endothelial cells (HRECs) inhibited the expression and deacetylase activity of SIRT1. The reduction of SIRT1 expression and deacetylase activity persisted following a return to normal glucose levels. Furthermore, nuclear factor- $\mathrm{\kappa B}$ expression was observed to be negatively correlated with SIRT1 expression and activity in HRECs under high glucose levels and the subsequent return to normal glucose levels. Fenofibrate treatment abrogated these changes. Knockdown of SIRT1 attenuated the effect of fenofibrate on high glucose-induced NF- $\mathrm{kB}$ expression. In addition, fenofibrate upregulated SIRT1 expression through peroxisome proliferator-activated receptor $\alpha$ in high glucose-induced metabolic memory. These findings indicate that fenofibrate is important in anti-inflammatory processes and suppresses the cellular metabolic memory of high glucose-induced stress via the SIRT1-dependent signalling pathway. Thus, treatment with fenofibrate may offer a
\end{abstract}

Correspondence to: Dr Zhi Zheng, Department of Ophthalmology, First People's Hospital of Shanghai, Shanghai Jiaotong University, 100 Haining Road, Shanghai 200080, P.R. China

E-mail: zzheng88@live.com

Key words: fenofibrate, sirtuin 1, nuclear factor- $\kappa \mathrm{B}$ cells, peroxisome proliferator-activated receptor $\alpha$, metabolic memory, diabetic retinopathy promising therapeutic strategy for halting the development of DR and other complications of diabetes.

\section{Introduction}

Diabetic retinopathy (DR) is the most common microvascular complication in diabetes, and DR has emerged as a leading cause of visual impairment and blindness in individuals aged $>50$ years (1). Clinical and experimental evidence has revealed that diabetic microvascular and macrovascular complications persist in diabetic patients regardless of whether blood glucose normalisation has occurred; this phenomenon has been defined as 'metabolic memory' (2-5). Although numerous studies have investigated the underlying mechanisms of metabolic memory (5), this particular negative phenomenon remains poorly understood and poses a major challenge in the treatment of diabetes.

There is accumulating evidence that DR exhibits certain characteristics of a low-grade inflammatory disease, in which retinal inflammatory mediators and apoptosis of retinal cells contribute to the process of metabolic memory (6-8). Nuclear factor $(\mathrm{NF})-\kappa \mathrm{B}$ is a master regulator of various genes involved in inflammatory and immune responses, cellular proliferation and apoptosis (9-11). Diabetes-induced activation of NF- $\mathrm{KB}$ was shown to promote expression of proinflammatory cytokines and various pro-apoptosis regulators (4). This activation also contributes to the apoptosis of retinal endothelial cells (RECs), which are significant in the pathogenesis of DR (12). Further studies have demonstrated that NF- $\mathrm{KB}$ is activated in the retina as early as two months after the onset of diabetes (12). Reinstitution of good blood glucose control after six months of poor blood glucose control did not exhibit an effect on activated NF- $\mathrm{kB}$ levels in the retinas of streptozotocin (STZ)-induced diabetic rats, indicating that NF- $\mathrm{KB}$-associated signalling pathways remained activated, resulting in a cellular metabolic memory effect (7). However, the mechanisms by which hyperglycaemia induces the activation of NF- $\mathrm{kB}$ and its dependent signalling pathways in diabetic metabolic memory have not been elucidated.

Class III histone deacetylase, sirtuin 1 (SIRT1) is a multifunctional deacetylase that is critically involved in regulating 
inflammation, stress responses, metabolism, DNA repair and cell survival via deacetylation of key transcription factors, enzymes and proteins (13-15). Our previous study demonstrated that SIRT1 conferred resistance to cellular metabolic memory, which had been induced by high glucose (5). Recently, both in vitro and in vivo studies indicated that SIRT1 suppresses $\mathrm{NF}-\kappa \mathrm{B}$ signalling and results in the reduction of the inflammatory responses mediated by $\mathrm{NF}-\kappa \mathrm{B}$ in endothelial cells $(16,17)$. Therefore, SIRT1 may be significant in the pathogenesis of the metabolic memory phenomenon via the $\mathrm{NF}-\kappa \mathrm{B}$ signalling pathway.

Fenofibrate, a peroxisome proliferator-activated receptor $\alpha$ $(\operatorname{PPAR} \alpha)$ agonist, is an effective lipid-lowering therapeutic agent that is widely administered in the clinical setting. In addition to its lipid effects, fenofibrate affects various signalling pathways involved in inflammation, angiogenesis and cell survival, and has received attention as a novel medical treatment for DR and other diabetes-induced microvascular complications. A previous study indicated that fenofibrate inhibits high glucose-induced metabolic memory in Schwann cells in diabetic neuropathy (18). Additional previous studies indicated that fenofibrate activates SIRT1 and suppresses cellular inflammation by activation of $\operatorname{PPAR} \alpha(19,20)$.

These findings resulted in the current investigation to establish whether fenofibrate may suppress high glucose-induced metabolic memory via its anti-inflammatory effect in endothelial cells during DR. Furthermore, the association between fenofibrate and the SIRT1-dependent signalling pathway was assessed.

\section{Materials and methods}

Cell culture and treatment. Human RECs (HRECs) and attachment factor were purchased from the Applied Cell Biology Research Institute (Kirkland, WA, USA) and maintained in EGM2-MV media (Lonza Group AG, Basel, Switzerland) with $5 \%$ fetal bovine serum (Lonza Inc., Allendale, NJ, USA) in flasks coated with the attachment factor. Cultured HRECs of three to four passages were used in the experiments. The cells were incubated with a normal concentration of glucose (normal glucose; $5 \mathrm{mmol} / \mathrm{l}$ ) for three weeks, a high concentration of glucose (high glucose; $30 \mathrm{mmol} / \mathrm{l}$ ) for 3 weeks, or high glucose for one week followed by normal glucose for two weeks.

For pharmacological prevention of glucose-induced metabolic memory, after 1 week, cells were switched from high glucose to normal glucose with fenofibrate at various concentrations $(25,50$ and $100 \mu \mathrm{M})$ for $48 \mathrm{~h}$ and subsequently maintained without fenofibrate for 12 days. To equalize the osmolarity and rule out the influence of increased osmolarity on the cellular memory of high glucose-induced stress, cells were incubated in $25 \mathrm{mmol} / \mathrm{l}$ mannitol along with normal glucose at $5 \mathrm{mmol} / \mathrm{l}$, which served as osmotic controls. Additionally, PPAR $\alpha$ antagonist, GW6471 $(1 \mu \mathrm{M})$ was added to the media for $1 \mathrm{~h}$ and incubated with fenofibrate to investigate whether the protective effect of fenofibrate on SIRT1 expression is mediated by PPAR $\alpha$ activation in diabetic metabolic memory.

RNA interference and transfection. The human small interfering (si)RNA targeting SIRT1 (SIRT1 siRNA) and negative control (NC) siRNA were chemically synthesized by Shanghai GenePharma Co., Ltd. (Shanghai, China) using the following sequences: 5'-GAUGCUGUGAAAUUACUGC-3' for SIRT1 siRNA and 5'-GGATCATAAGGCGCATAGC-3' for NC siRNA. Transfection was performed with Lipofectamine 2000 reagent (Invitrogen Life Technologies, Carlsbad, CA, USA) according to the manufacturer's instructions. A final concentration of $50 \mathrm{nM}$ RNA (for SIRT1 siRNA) or 100 nM RNA (for NC siRNA) and their respective NCs was used for each transfection in the subsequent experiments.

Quantitative polymerase chain reaction ( $q P C R)$. Total RNA was extracted from cultured cells using TRIzol reagent (Invitrogen Life Technologies). The expression level of SIRT1 mRNA was quantified by qPCR using a QuantiTect SYBR ${ }^{\circledR}$ Green PCR kit (Qiagen GmbH, Hilden, Germany) and normalized to $\beta$-actin using the following primers: Forward 5'-AGTACTGGGGAGAAAAATGAAAGA-3' and reverse 5'-CTGCCACAAGAACTAGAGGATAAG-3' for SIRT1; forward 5'-CCCAAGGCCAACCGCGAGAAGATG-3' and reverse 5'-GTCCCGGCCAGCCAGGTCCAGA-3' for $\beta$-actin. The cycling conditions were as follows: Denaturation at $95^{\circ} \mathrm{C}$ for $10 \mathrm{sec}$, followed by annealing at $58^{\circ} \mathrm{C}$ for $20 \mathrm{sec}$ and extension at $58^{\circ} \mathrm{C}$ for $20 \mathrm{sec}$, for 40 cycles. The changes in expression were calculated using the $2^{-\Delta \Delta \mathrm{Ct}}$ method (21).

Western blot analysis. Total protein was extracted from the cultured cells or tissues using a Total Protein Extraction kit (cat. no. 2140; EMD Millipore, Billerica, MA, USA) according to the manufacturer's instructions. The protein content was determined using a Bicinchoninic Acid Protein Assay kit (cat. no. 23225; Invitrogen Life Technologies) with bovine serum albumin (Gibco Life Technologies, Carlsbad, CA, USA) serving as the standard. Proteins $(20 \mu \mathrm{g})$ were separated by $10 \%$ SDS-PAGE and transferred onto polyvinylidene difluoride membranes (EMD Millipore). The membranes were blocked in 5\% non-fat milk and Tris-buffered saline with $0.05 \%$ Tween-20 (Invitrogen Life Technologies) at room temperature for $2 \mathrm{~h}$, then probed with antibodies as follows: Mouse anti-SIRT1 monoclonal antibody [1:6,000; Abcam, Cambridge, MA, USA (cat. no. ab110304)], rabbit anti-PPAR $\alpha$ polyclonal antibody [1:1,000; Santa Cruz Biotechnology, Dallas, TX, USA (cat. no. sc-2772)], human anti-NF-кB antibody [1:400; Enzo Life Sciences, Inc., Farmingdale, NY, USA (cat. no. 7971)] or $\beta$-actin [1:1,000, Sigma-Aldrich, St. Louis, MO, USA (cat. no. A2103)] and developed with an enhanced chemiluminescence kit (cat. no. RPN2132; GE Healthcare Life Sciences, Chalfont, UK).

SIRT1 deacetylase activity assay. SIRT1 deacetylase activity was assessed in the nuclear fraction using a commercial fluorometric assay kit (cat. no. CS1040; Sigma-Aldrich). Protein (30-40 $\mu \mathrm{g}$ ) was incubated with the substrate (coupled to the fluorophore and quencher) and $\mathrm{NAD}^{+}$for $3 \mathrm{~min}$ at room temperature. The fluorescence emitted, due to deacetylation of the substrate by SIRT1, was measured at $345 \mathrm{~nm}$ excitation and $450 \mathrm{~nm}$ emission wavelengths using a fluorescence microplate reader (SpectraMax ${ }^{\circledR}$ M5; Molecular Devices, LLC, Sunnyvale, CA, USA). 
A

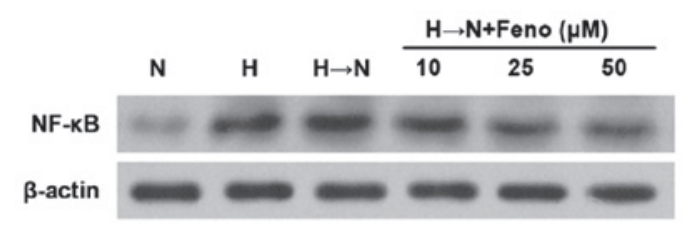

C

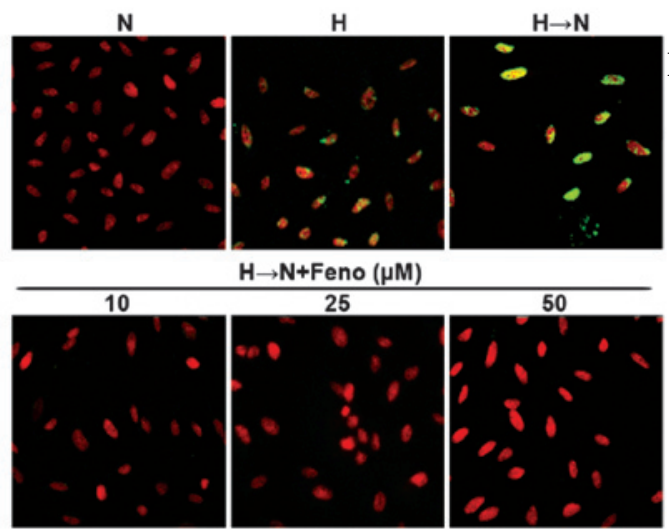

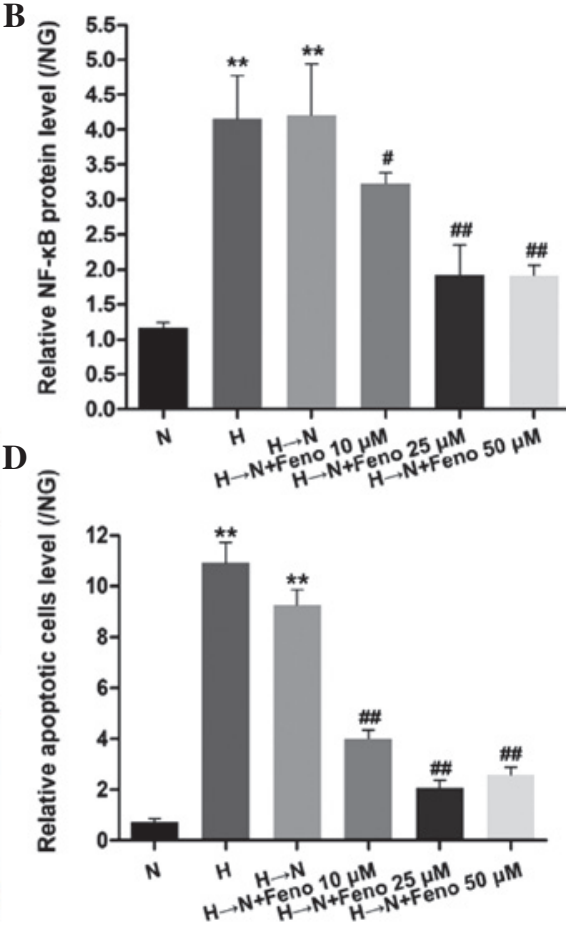

Figure 1. Feno reversed the increased expression of $\mathrm{NF}-\kappa \mathrm{B}$ and cellular apoptosis in human retinal vascular endothelial cells following culture in a high concentration of glucose followed by a normal concentration of glucose. (A) Western blotting and (B) quantification of NF- $\kappa \mathrm{B}$ protein expression profiles in the cell treatment groups: Normal glucose $(\mathrm{N})$, high glucose $(\mathrm{H})$, high glucose followed by normal glucose $(\mathrm{H} \rightarrow \mathrm{N})$, and $\mathrm{H} \rightarrow \mathrm{N}$ plus Feno at different concentrations $(10,25$ and $50 \mu \mathrm{M})$. (C) Analysis of cellular apoptosis levels conducted in the six groups by TUNEL assay. (D) Analysis of apoptotic cell number. (B,D) Results are presented as the means + standard deviations of three representative experiments. The results of all the groups are displayed as the ratio of the control, NG (normal glucose). ${ }^{* *} \mathrm{P}<0.01$ vs. $\mathrm{N} ;{ }^{~} \mathrm{P}<0.05$ vs. $\mathrm{H} \rightarrow \mathrm{N}$; ${ }^{\# \#} \mathrm{P}<0.01$ vs. $\mathrm{H} \rightarrow \mathrm{N}$. Feno, fenofibrate; NF- $\mathrm{B}$, nuclear factor- $\kappa \mathrm{B}$.

TUNEL assay. Cells treated with normal glucose, high glucose, high glucose followed by normal glucose, or high glucose followed by normal glucose plus fenofibrate at different concentrations $(10,25$ and $50 \mu \mathrm{M})$ were grown on glass coverslips in 24-well plates. The cells were then fixed with $4 \%$ paraformaldehyde, which was followed by permeabilization with $0.1 \%$ Triton X-100 (Sigma-Aldrich). The apoptotic cells were stained with a fluorometric TUNEL assay kit (DeadEnd ${ }^{\mathrm{TM}}$ Fluorometric TUNEL System; Promega Corporation, Madison, WI, USA) and visualized under a fluorescence microscope (Olympus BX41; Olympus Corporation, Tokyo, Japan) according to the manufacturer's instructions. TUNEL-positive cells were scored in a minimum of five fields per coverslip, and $\geq 1,000$ cells were counted for each coverslip.

Statistical analysis. Data were presented as the mean \pm standard deviation from at least three independent experiments. Group means were compared by one-way analysis of variance using GraphPad Prism 4.0 software system (GraphPad, San Diego, CA, USA) and the statistical software program, SPSS version 17.0 (SPSS, Inc., Chicago, IL, USA). Correlations between $\mathrm{NF}-\kappa \mathrm{B}$ expression and the expression and activity of SIRT1 were calculated using Spearman's rank correlation. All $\mathrm{P}$-values were two-sided and $\mathrm{P}<0.05$ was considered to indicate a statistically significant difference.

\section{Results}

Fenofibrate suppressed the expression of $N F-\kappa B$ induced by high glucose after glucose normalization in HRECs.
To investigate the inhibitory effect of fenofibrate on $\mathrm{NF}-\kappa \mathrm{B}$ expression in high glucose-induced metabolic memory in HRECs, the cells were exposed to normal $(5 \mathrm{mmol} / \mathrm{l})$ or high (30 mmol/l) glucose concentrations, or high glucose followed by normal glucose with different concentrations of fenofibrate $(10,25$ and $50 \mu \mathrm{M})$.

As shown in Fig. 1A and B, chronic exposure to high glucose resulted in significantly increased protein levels of $\mathrm{NF}-\kappa \mathrm{B}$. Compared with exposure to continuous normal glucose, $\mathrm{NF}-\kappa \mathrm{B}$ remained increased in the cells that were treated with high glucose for 1 week followed by normal glucose for 2 weeks. Fenofibrate downregulated the protein expression of $\mathrm{NF}-\kappa \mathrm{B}$, indicating that fenofibrate suppresses high glucose-induced NF- $\mathrm{NB}$ expression following glucose normalization in HRECs.

To equalize the osmolarity with the high glucose treatment at $30 \mathrm{mmol} / 1$ and rule out the influence of increased osmolarity on the cellular memory of high glucose-induced stress, $25 \mathrm{mmol} / \mathrm{l}$ mannitol was added along with $5 \mathrm{mmol} / \mathrm{l}$ normal glucose to cells for 3 weeks, and no effects on memory were observed (data not shown).

Furthermore, the level of cellular apoptosis was observed using a TUNEL assay. Chronic exposure to high glucose caused a significant increase in cellular apoptosis. Apoptosis also remained increased in the cells exposed to high glucose for 1 week followed by normal glucose for 2 weeks. Fenofibrate suppressed the cellular apoptosis in high glucose-induced metabolic memory in HRECs (Fig. 1C and D). These results support the hypothesis that NF- $\kappa \mathrm{B}$ is involved in fenofibrate's suppression of high glucose-induced cellular metabolic memory. 

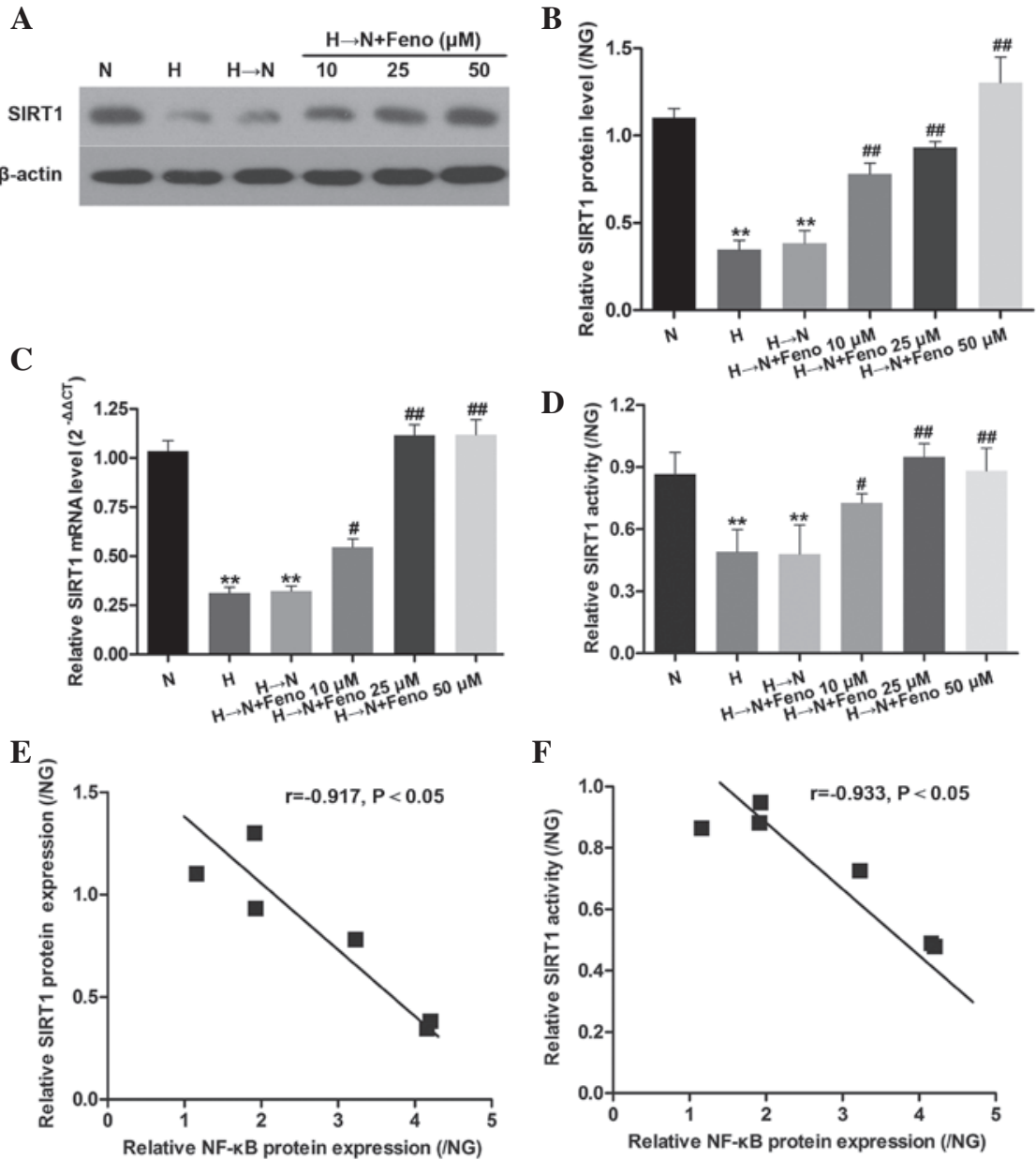

Figure 2. Inverse correlation between NF- $\mathrm{KB}$ and SIRT1 protein expression and activity levels in HRECs. (A) Relative expression of SIRT1 mRNA in the cell treatment groups: Normal glucose $(\mathrm{N})$, high glucose $(\mathrm{H})$, high glucose followed by normal glucose $(\mathrm{H} \rightarrow \mathrm{N})$, and $\mathrm{H} \rightarrow \mathrm{N}$ plus Feno at different concentrations $(10,25$ and $50 \mu \mathrm{M})$. SIRT1 (B) protein, (C) mRNA and (D) activity levels in the six HRECs groups. Results are presented as the means + standard deviations of three representative experiments. (E and F) Inverse correlation was calculated by Spearman's correlation: (E) r=-0.523 (P=0.018) for NF- $\mathrm{kB}$ and SIRT1 protein expression and $(\mathrm{F}) \mathrm{r}=-0.397(\mathrm{P}=0.043)$ for NF- $\mathrm{\kappa B}$ and SIRT1 activity level. The results of all the groups are displayed as the ratio of the control, NG (normal glucose). ${ }^{* *} \mathrm{P}<0.01$ vs. $\mathrm{N} ;{ }^{*} \mathrm{P}<0.05$ vs. $\mathrm{H} \rightarrow \mathrm{N} ;{ }^{\# \#} \mathrm{P}<0.01 \mathrm{vs}$. $\mathrm{H} \rightarrow \mathrm{N}$. The relative expression data were analyzed using the $2^{-\Delta \Delta C t}$ method. SIRT1, sirtuin 1 ; HRECs, human retinal vascular endothelial cells; Feno, fenofibrate; NF- $\kappa B$, nuclear factor- $\kappa B$.

Fenofibrate inhibits high glucose-induced $N F-\kappa B$ expression through SIRT1 in HRECs. To investigate the potential synergistic roles of SIRT1, NF- $\kappa$ B and fenofibrate in modulating high glucose-induced metabolic memory in HRECs, qPCR and western blot analysis were conducted to examine the expression levels of SIRT1 in the cells exposed to normal glucose, high glucose, or high glucose followed by normal glucose with different concentrations of fenofibrate $(10,25$ and $50 \mu \mathrm{M})$. As shown in Fig. 2A-D, chronic exposure to high glucose resulted in significantly decreased levels of SIRT1. SIRT1 levels remained decreased in cells treated with high glucose for 1 week followed by normal glucose for 2 weeks when compared with exposure to continuous normal glucose. However, fenofibrate significantly suppressed the inhibition of SIRT1, which was induced by high glucose following glucose normalization in HRECs. Furthermore, a significant negative correlation between NF- $\mathrm{KB}$ and SIRT1 protein expression and activity levels was revealed in HRECs (Fig. 2E and F).

To confirm the regulatory role of SIRT1 in fenofibrate-mediated inhibition of NF- $\mathrm{KB}$ expression, HRECs were transfected with SIRT1-specific siRNA to decrease SIRT1 expression prior to incubation with fenofibrate. The results showed that the inhibitory effect of fenofibrate on high glucose-induced NF- $\mathrm{BB}$ protein expression and cellular apoptosis was abolished by knockdown of SIRT1 (Fig. 3). Therefore, these results indicate that fenofibrate inhibits high glucose-induced metabolic memory in HRECs via SIRT1-dependent suppression of NF- $\kappa$ B.

Fenofibrate upregulates SIRT1 expression through PPARQ activation in HRECs. To investigate whether the protective effect of fenofibrate on SIRT1 expression is mediated by PPAR $\alpha$ activation in diabetic metabolic memory, HRECs were pretreated with the PPAR $\alpha$ antagonist, GW6471 $(1 \mu \mathrm{M})$ for $1 \mathrm{~h}$ and incubated with fenofibrate. As shown in Fig. 4, chronic exposure to high glucose decreased the expression of PPAR $\alpha$ even after glucose normalization in HRECs. Pretreatment of the cells with fenofibrate increased the PPAR $\alpha$ expression and this effect was abolished by treatment with GW6471. Furthermore, pretreating the cells with $1 \mu \mathrm{M}$ GW6471 reversed the effect of fenofibrate on SIRT1 protein expression. These 

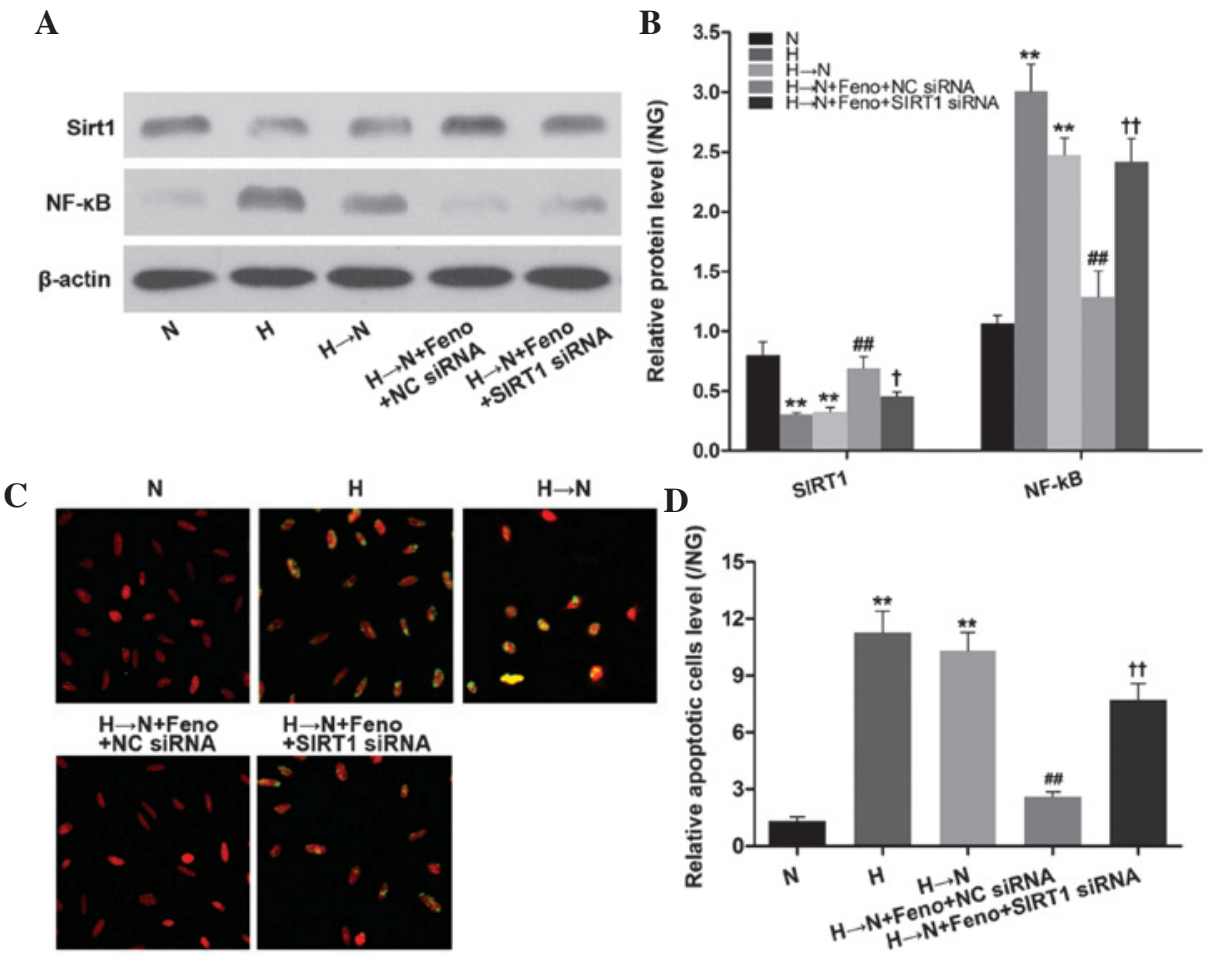

Figure 3. Feno inhibits NF-kB expression and cellular apoptosis through SIRT1 in human retinal vascular endothelial cells following culture in high glucose followed by normal glucose $(\mathrm{H} \rightarrow \mathrm{N})$. (A) Western blotting and (B) quantification of SIRT1 and NF- $\mathrm{kB}$ protein expression in the cell treatment groups: Normal glucose $(\mathrm{N})$, high glucose $(\mathrm{H})$, high glucose followed by normal glucose $(\mathrm{H} \rightarrow \mathrm{N}), \mathrm{H} \rightarrow \mathrm{N}$ plus Feno and NC siRNA $(\mathrm{H} \rightarrow \mathrm{N}+\mathrm{Feno}+\mathrm{NC}$ siRNA) or SIRT1 siRNA $(\mathrm{H} \rightarrow \mathrm{N}+$ Feno + SIRT1 siRNA). (C) Analysis of cellular apoptosis levels conducted in the five groups by TUNEL. (D) Analysis of apoptotic cell number. (B,D) Results are presented as the means + standard deviations of three representative experiments. The results of all the groups are displayed as the ratio of the

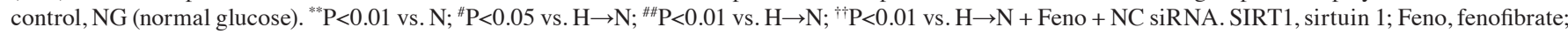
$\mathrm{NF}-\kappa \mathrm{B}$, nuclear factor- $\kappa \mathrm{B} ; \mathrm{NC}$, negative control; siRNA, small interfering RNA.

A

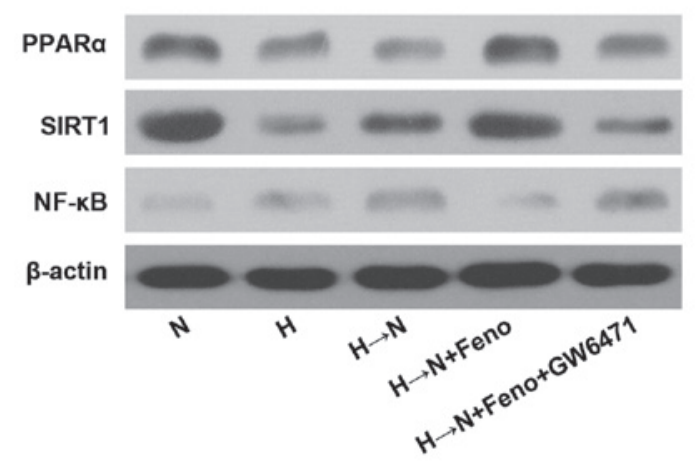

B

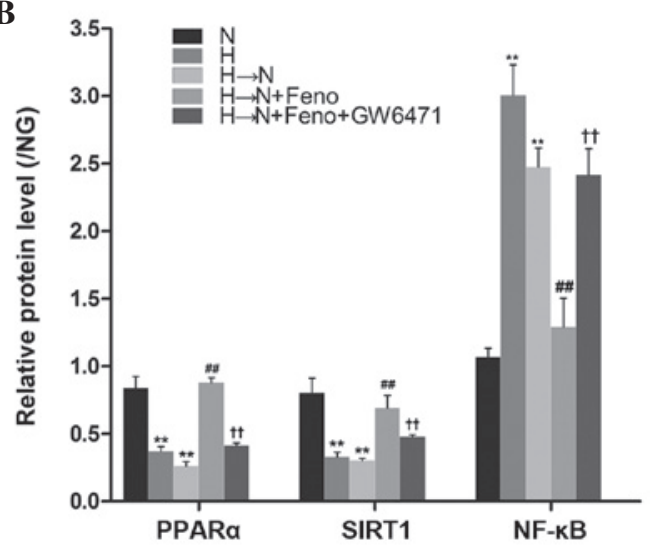

Figure 4. Feno upregulates SIRT1 expression through PPAR $\alpha$ activation in human retinal vascular endothelial cells. (A) Western blotting and (B) quantification of SIRT1 and NF- $\mathrm{KB}$ protein expression in the five cell treatment groups: Normal glucose $(\mathrm{N})$, high glucose $(\mathrm{H})$, high glucose followed by normal glucose $(\mathrm{H} \rightarrow \mathrm{N}), \mathrm{H} \rightarrow \mathrm{N}$ plus Feno $(\mathrm{H} \rightarrow \mathrm{N}+\mathrm{Feno})$ and $\mathrm{H} \rightarrow \mathrm{N}$ plus Feno and PPAR $\alpha$ antagonist, GW6471 $(\mathrm{H} \rightarrow \mathrm{N}+$ Feno + GW6471). Results are presented as the means + standard deviations of three representative experiments. The results of all the groups are displayed as the ratio of the control, NG (normal glucose). ${ }^{* *} \mathrm{P}<0.01$ vs. $\mathrm{N} ;{ }^{\#} \mathrm{P}<0.05$ vs. $\mathrm{H} \rightarrow \mathrm{N}$; ${ }^{\# \#} \mathrm{P}<0.01$ vs. $\mathrm{H} \rightarrow \mathrm{N} ;{ }^{\dagger} \mathrm{P}<0.01$ vs. $\mathrm{H} \rightarrow \mathrm{N}+$ Feno. Feno, fenofibrate; SIRT1, sirtuin 1; PPAR $\alpha$, peroxisome proliferator-activated

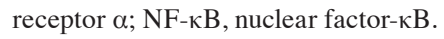

results indicate that fenofibrate upregulates SIRT1 expression through activation of PPAR $\alpha$ in HRECs.

\section{Discussion}

Our previous study demonstrated that SIRT1 confers resistance to cellular metabolic memory induced by high glucose (5). In the current study, further evidence is presented that fenofibrate upregulates SIRT1 expression and activity via PPAR $\alpha$ activation, and downregulates $\mathrm{NF}-\kappa \mathrm{B}$ expression to suppress the memory of hyperglycaemic stress in HRECs.

A major challenge in treating diabetic microvascular complications, such as DR, is that the molecular and pathological features resulting from high glucose are maintained despite subsequent effective control of blood glucose $(22,23)$. The prolonged impact of the early metabolic environment on 
the development and progression of microvascular complications is referred to as 'metabolic memory' (24).

Inflammation is significant early and throughout the pathogenesis of microvascular complications (25). NF- $\kappa \mathrm{B}$ is a rapid response transcription factor involved in inflammatory reactions, as well as the expression of cytokines, chemokines, cell adhesion molecules and growth factors. It is considered to be a key signalling factor by which high glucose concentrations trigger a pro-apoptotic program in diabetes (26). A study by Kowluru et al (7) using STZ-induced diabetic rats indicated that chronic exposure to high glucose caused a significant increase in the levels of activated caspase -3 and $N F-\kappa B$, which remained at the increased levels six months later (7), suggesting that the activated inflammation-associated signalling pathways had remained activate. In our previous study (5), using an established cell model of metabolic memory induced by high glucose, the reinstitution of normal glucose levels after 1 week of high glucose was observed to have no effect on the levels of activated $\mathrm{NF}-\kappa \mathrm{B}$ expression and cell apoptosis. This finding indicated that the activated inflammation-associated signalling pathways remained activated.

There is accumulating evidence that SIRT1 inhibits NF- $\kappa \mathrm{B}$ signalling, and the activation of SIRT1 may alleviate a multitude of NF- $\kappa \mathrm{B}$-driven inflammatory and metabolic disorders (27-29). In contrast to NF- $\mathrm{B}$, the present study showed an adverse tendency of SIRT1 expression in HREC cells. The results were consistent with our previous study, which demonstrated that SIRT1 activation reduced high glucose-induced cellular metabolic memory in RECs by suppressing production of the cellular inflammatory gene, $N F-\kappa B$ and attenuating the expression of the cellular apoptosis gene, Bax (5). These data implied that SIRT1 activators may exert significant protective effects against metabolic memory in diabetic microvascular complications, such as DR.

Recently, fenofibrate, a specific PPAR $\alpha$ agonist, has displayed marked and robust efficacy in arresting the progression of microvascular complications in type 2 diabetes in FIELD and ACCORD studies (30,31). Furthermore, various studies have demonstrated that fenofibrate activates SIRT1 and suppresses cellular inflammation by activation of PPAR $\alpha(19,20)$. However, its function in the retina has rarely been investigated. In the present study, it was found that fenofibrate dose-dependently reversed the changes to SIRT1 and $\mathrm{NF}-\kappa \mathrm{B}$ expression in high glucose-induced cellular metabolic memory in HRECs. Knockdown of SIRT1 attenuated the inhibitory effect of fenofibrate on NF- $\mathrm{NB}$ expression, suggesting that fenofibrate inhibits high glucose-induced metabolic memory in HRECs via SIRT1-dependent suppression of NF- $\kappa \mathrm{B}$.

A recent study found that the inhibitory effect of SIRT1 on monocyte chemoattractant protein-1 mRNA expression was attenuated by the PPAR $\alpha$ antagonist, GW6471 in cardiomyocytes, indicating that SIRT1 acted in association with PPAR $\alpha$ to protect cardiomyocytes from inflammation (32). Thus, whether the protective effect of fenofibrate on SIRT1 expression was mediated by PPAR $\alpha$ activation in diabetic metabolic memory was investigated in the current study. It was demonstrated that exposure to high glucose levels reduced PPAR $\alpha$ expression, whereas treatment with fenofibrate activated PPAR $\alpha$ and exerted an anti-inflammatory effect via SIRT1-dependent suppression of $\mathrm{NF}-\kappa \mathrm{B}$.
Various studies indicate that fenofibrate may inhibit $\mathrm{NF}-\kappa \mathrm{B}-$ mediated cellular inflammation by suppressing the AMPK/eNOS/NO $(33,34)$ and Toll-like receptor signalling pathways (35). However, the mechanism by which fenofibrate may inhibit NF- $\kappa \mathrm{B}$ signalling pathways in diabetic microvascular dysfunction remains unclear. To the best of our knowledge, the present study is the first to link SIRT1 with the inhibitory effect of fenofibrate on NF- $\kappa \mathrm{B}$-mediated cellular inflammation in high glucose-induced cellular metabolic memory. Notably, a recent study indicated that the PPAR $\alpha$ agonist, fenofibrate inhibits tumour necrosis factor $\alpha$-induced cluster of differentiation 40 expression and regulates the inflammatory response in 3T3-L1 adipocytes via the SIRT1-dependent signalling pathway (20). Our results are consistent with this, and support the hypothesis that PPAR $\alpha / \mathrm{SIRT} 1 / \mathrm{NF}-\kappa \mathrm{B}$ may be a commonly used signalling pathway during the cellular inflammation of different pathological processes.

In conclusion, the present study demonstrates that high glucose levels activate the $\mathrm{NF}-\kappa \mathrm{B}$-associated inflammation signalling pathway and induce metabolic memory, which prolong the impact of early metabolic dysfunction on the progression of retinal endothelial injury. Fenofibrate was observed to activate PPAR $\alpha$ and inhibit high glucose-induced metabolic memorial injury via SIRT1-dependent suppression of NF- $\kappa \mathrm{B}$ in HRECs. These findings may provide a promising strategy for suppressing the development of DR and other associated complications of diabetes.

\section{Acknowledgements}

The present study was supported by grants from the National Natural Science Foundation of China (grant nos. 81271032, 81070739 and 30872828) and the National Key Basic Research Programme (grant nos. 2010CB535006).

\section{References}

1. Lutty GA: Effects of diabetes on the eye. Invest Ophthalmol Vis Sci 54: ORSF81-87, 2013.

2. No authors listed: Retinopathy and nephropathy in patients with type 1 diabetes four years after a trial of intensive therapy. The Diabetes Control and Complications Trial/Epidemiology of Diabetes Interventions and Complications Research Group. N Engl J Med 342: 381-389, 2000.

3. Writing Team for the Diabetes Control and Complications Trial/Epidemiology of Diabetes Interventions and Complications Research Group: Effect of intensive therapy on the microvascular complications of type 1 diabetes mellitus. JAMA 287: 2563-2569, 2002.

4. Ceriello A, Ihnat MA and Thorpe JE: Clinical review 2: The 'metabolic memory': is more than just tight glucose control necessary to prevent diabetic complications? J Clin Endocrinol Metab 94: 410-415, 2009.

5. Zheng Z, Chen H, Li J, Li T, Zheng B, Zheng Y, Jin H, He Y, $\mathrm{Gu} \mathrm{Q}$ and $\mathrm{Xu} \mathrm{X}$ : Sirtuin 1-mediated cellular metabolic memory of high glucose via the LKB1/AMPK/ROS pathway and therapeutic effects of metformin. Diabetes 61: 217-228, 2012.

6. Kowluru RA and Chan PS: Metabolic memory in diabetes - from in vitro oddity to in vivo problem: Role of apoptosis. Brain Res Bull 81: 297-302, 2010.

7. Kowluru RA, Chakrabarti S and Chen S: Re-institution of good metabolic control in diabetic rats and activation of caspase- 3 and nuclear transcriptional factor (NF-kappaB) in the retina. Acta Diabetol 41: 194-199, 2004.

8. Kowluru RA, Zhong Q and Kanwar M: Metabolic memory and diabetic retinopathy: Role of inflammatory mediators in retinal pericytes. Exp Eye Res 90: 617-623, 2010. 
9. Sen R and Baltimore D: Inducibility of kappa immunoglobulin enhancer-binding protein Nf-kappa B by a posttranslational mechanism. Cell 47: 921-928, 1986.

10. Oeckinghaus A and Ghosh S: The NF-kappaB family of transcription factors and its regulation. Cold Spring Harb Perspect Biol 1: a000034, 2009.

11. DiDonato JA, Mercurio F and Karin M: NF-kappaB and the link between inflammation and cancer. Immunol Rev 246: 379-400, 2012.

12. Kowluru RA, Koppolu P, Chakrabarti S and Chen S Diabetes-induced activation of nuclear transcriptional factor in the retina and its inhibition by antioxidants. Free Radic Res 37 $1169-1180,2003$.

13. Yu J and Auwerx J: Protein deacetylation by SIRT1: An emerging key post-translational modification in metabolic regulation. Pharmacol Res 62: 35-41, 2010.

14. Haigis MC and Sinclair DA: Mammalian sirtuins: Biological insights and disease relevance. Annu Rev Pathol 5: 253-295, 2010.

15. Baur JA: Resveratrol, sirtuins and the promise of a DR mimetic Mech Ageing Dev 131: 261-269, 2010.

16. Cai W, Ramdas M, Zhu L, Chen X, Striker GE and Vlassara H: Oral advanced glycation endproducts (AGEs) promote insulin resistance and diabetes by depleting the antioxidant defenses AGE receptor-1 and sirtuin 1. Proc Natl Acad Sci USA 109: 15888-15893, 2012.

17. Tsai KL, Huang PH, Kao CL, Leu HB, Cheng YH, Liao YW, Yang YP, Chien Y, Wang CY, Hsiao CY, et al: Aspirin attenuates vinorelbine-induced endothelial inflammation via modulating SIRT1/AMPK axis. Biochem Pharmacol 88: 189-200, 2014.

18. Kim ES, Isoda F, Kurland I and Mobbs CV: Glucose-induced metabolic memory in Schwann cells: prevention by PPAR agonists. Endocrinology 154: 3054-3066, 2013.

19. Wang W, Bai L, Qiao H, Lu Y, Yang L, Zhang J, Lin R, Ren F, Zhang J and Ji M: The protective effect of fenofibrate against TNF- $\alpha$-induced CD40 expression through SIRT1-mediated deacetylation of NF- $\mathrm{B}$ in endothelial cells. Inflammation 37: 177-185, 2014.

20. Wang W, Lin Q, Lin R, Zhang J, Ren F, Zhang J, Ji M and Li Y: PPARalpha agonist fenofibrate attenuates TNF- $\alpha$-induced CD40 expression in 3T3-L1 adipocytes via the SIRT1-dependent signaling pathway. Exp Cell Res 319: 1523-1533, 2013.

21. Livak KJ and Schmittgen TD: Analysis of relative gene expression data using real-time quantitative PCR and the 2(-Delta Delta C(T)) Method. Methods 25: 402-408, 2001.

22. No authors listed: The effect of intensive diabetes therapy on the development and progression of neuropathy. The Diabetes Control and Complications Trial Research Group. Ann Intern Med 122: 561-568, 1995.
23. Writing Team for the Diabetes Control and Complications Trial/Epidemiology of Diabetes Interventions and Complications Research Group: Sustained effect of intensive treatment of type 1 diabetes mellitus on development and progression of diabetic nephropathy: the Epidemiology of Diabetes Interventions and Complications (EDIC) study. JAMA 290: 2159-2167, 2003.

24. Ihnat MA, Thorpe JE and Ceriello A: Hypothesis: The 'metabolic memory', the new challenge of diabetes. Diabet Med 24: 582-586, 2007.

25. LeRoith D, Fonseca V and Vinik A: Metabolic memory in diabetes-focus on insulin. Diabetes Metab Res Rev 21: 85-90, 2005.

26. Baker RG, Hayden MS and Ghosh S: NF-кB, inflammation and metabolic disease. Cell Metab 13: 11-22, 2011.

27. Salminen A, Kauppinen A, Suuronen T and Kaarniranta K: SIRT1 longevity factor suppresses NF-kappaB-driven immune responses: Regulation of aging via NF-kappaB acetylation? Bioessays 30: 939-942, 2008.

28. Yao $\mathrm{H}$ and Rahman I: Perspectives on translational and therapeutic aspects of SIRT1 in inflammaging and senescence. Biochem Pharmacol 84: 1332-1339, 2012.

29. Xie J, Zhang $X$ and Zhang L: Negative regulation of inflammation by SIRT1. Pharmacol Res 67: 60-67, 2013.

30. Keech AC, Mitchell P, Summanen PA, O'Day J, Davis TM, Moffitt MS, Taskinen MR, Simes RJ, Tse D, Williamson E, et al: Effect of fenofibrate on the need for laser treatment for diabetic retinopathy (FIELD study): A randomised controlled trial. Lancet 370: 1687-1697, 2007.

31. Ginsberg HN, Elam MB, Lovato LC III, Crouse JR III, Leiter LA, Linz P, Friedewald WT, Buse JB, Gerstein HC, Probstfield J, et al: Effects of combination lipid therapy in type 2 diabetes mellitus. N Engl J Med 362: 1563-1574, 2010.

32. Planavila A, Iglesias R, Giralt M and Villarroya F: Sirt1 acts in association with PPARalpha to protect the heart from hypertrophy, metabolic dysregulation and inflammation. Cardiovasc Res 90: 276-284, 2011

33. Biscetti F, Gaetani E, Flex A, Aprahamian T, Hopkins T, Straface G, Pecorini G, Stigliano E, Smith RC, Angelini F, et al: Selective activation of peroxisome proliferator-activated receptor (PPAR)alpha and PPAR gamma induces neoangiogenesis through a vascular endothelial growth factor-dependent mechanism. Diabetes 57: 1394-1404, 2008.

34. Okayasu T, Tomizawa A, Suzuki K, Manaka K and Hattori Y: PPARalpha activators upregulate eNOS activity and inhibit cytokine-induced NF-kappaB activation through AMP-activated protein kinase activation. Life Sci 82: 884-891, 2008.

35. Shen W, Gao Y, Lu B, Zhang Q, Hu Y and Chen Y: Negatively regulating TLR4/NF-kappaB signaling via PPAR $\alpha$ in endotoxin-induced uveitis. Biochim Biophys Acta 1842: 1109-1120, 2014. 